\title{
Au croisement des logiques politiques et médicales : les « patients-camarades » des services psychiatriques de la Charité
}

(Berlin-Est, République démocratique allemande, 1959-1964)

At the crossroads of political and medical principles: "comrade patients" in the psychiatric services at the Charité Hospital (East Berlin, German Democratic

Republic, 1959-1964)

\section{Fanny Le Bonhomme}

\section{(2) OpenEdition}

\section{Journals}

Édition électronique

URL : http://journals.openedition.org/conflits/18704

DOI : $10.4000 /$ conflits. 18704

ISSN : $1777-5345$

Éditeur :

CCLS - Centre d'études sur les conflits lilberté et sécurité, L'Harmattan

Édition imprimée

Date de publication : 15 octobre 2013

Pagination : 21-38

ISBN : 978-2-343-01909-3

ISSN : $1157-996 \mathrm{X}$

\section{Référence électronique}

Fanny Le Bonhomme, «Au croisement des logiques politiques et médicales : les « patients-

camarades » des services psychiatriques de la Charité », Cultures \& Conflits [En ligne], 90 | été 2013

mis en ligne le 15 octobre 2014, consulté le 30 mars 2021. URL : http://journals.openedition.org/

conflits/18704; DOI : https://doi.org/10.4000/conflits.18704 


\title{
Au croisement des logiques politiques et médicales : les "patients-camarades» des services psychiatriques de la Charité
}

(Berlin-Est, République démocratique allemande, 1959-1964)

\section{Fanny LE BONHOMME}

Fanny Le Bonhomme est Doctorante (CERHIO, Rennes 2 / Potsdam - Centre Marc Bloch, Berlin). Elle prépare une thèse d'histoire sous la direction de Thomas Lindenberger (Zentrum für Zeithistorische Forschung, Potsdam) et Jacqueline Sainclivier (CERHIO, Rennes 2).

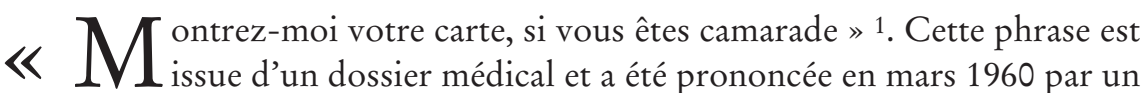
patient de la clinique psychiatrique et neurologique de la Charité (Berlin-Est, RDA). Le terme de «camarade » renvoyant à l'appellation des membres du parti communiste, elle traduit la volonté de ce patient de connaître l'appartenance politique du soignant qui lui fait face. Ce jour-là, L.R. refuse l'administration d'un soin, il demande à voir la carte du parti de son interlocuteur, menace de se plaindre au Comité central et exige qu'on aille chercher un "médecin-camarade » 2 . Ce faisant, il active son capital politique (il appartient lui-même - et de longue date - au parti communiste) et fait donc passer la logique politique avant la logique médicale. Cette irruption du politique dans les murs d'un service psychiatrique peut tout d'abord susciter un certain étonnement. Cet événement est d'ailleurs suffisamment inhabituel pour que le rédacteur juge nécessaire de le consigner dans le dossier afin d'illustrer cet accès d'agressivité. On est spontanément frappé par le croisement des logiques politiques et médicales, qui se noue dans la volonté du patient de se faire soigner par un « médecin-camarade». Un travail de contextualisation peut
\end{abstract}

1. «Zeigen Sie mir Ihren Ausweis, daß Sie Genossen sind. Ich werde mich beim ZK beschweren. Holen Sie sofort Herrn Dr H. » HPAC [Historisches Psychiatriearchiv der Charité], M, 116 / 60. Krankengeschichte, Verlauf, 3.3.60. [Traduction de l'auteur]

2. Les initiales des patients ont été anonymisées. 
cependant permettre de dépasser cet étonnement premier pour tenter d'expliquer cette réaction a priori inadéquate. On fait alors appel aux multiples « contexte[s] [...] qui lui donne[nt] sens et forme ${ }^{3}$.

Il convient tout d'abord de replacer cet incident dans le contexte général de la RDA, qui appartient, depuis sa fondation en 1949, à l'Europe communiste. La réalité du pouvoir y est concentrée entre les mains du parti communiste (SED), dont le projet politique de transformation sociale vise à l'avènement d'une "société socialiste " ${ }^{4}$. L'espace social de la RDA - en tant que structure de distribution des capitaux - possède une caractéristique commune aux sociétés de type soviétique : le capital économique y est officiellement mis hors jeu tandis que le capital politique se voit hissé au premier plan et peut apparaître comme un "nouveau principe orchestrateur » 5. Appartenir au parti communiste permet d'être identifié comme un partisan du régime, mais également d'accéder aux postes de responsabilité dans le pays. Un référentiel identitaire qui sépare les camarades des autres joue à plein dans le fonctionnement interne de la société est-allemande ${ }^{6}$. Le capital politique est donc un élément important pour rendre compte de la distribution des pouvoirs et des privilèges au sein de l'espace social, ce qui explique en partie la réaction de ce patient. A une autre échelle, son expérience biographique permet également d'éclairer l'incident. Né en 1904, maçon de formation, L.R. est engagé en politique depuis plus de 25 ans. Il a appartenu au parti communiste allemand (KPD) avant la création du SED en $1946^{7}$. C'est donc un communiste de la première génération. Son expérience politique s'est forgée pendant la période nazie, au cours de laquelle il a passé six ans en camp de concentration. Après 1945, il connaît une promotion sociale significative puisqu'il devient maire et travaille ensuite au contrôle gouvernemental. Dans cette situation conflictuelle qui l'oppose à l'équipe soignante, il n'accorde pas d'autre légitimité que celle qui l'a accompagné une grande partie de son existence et que confère uniquement le parti. Son parcours politique constitue bien un facteur supplémentaire pour expliquer sa volonté d'aller chercher un autre médecin. S'affirmant « camarade ", L.R. saisit cette occasion pour contourner son rôle de patient, afin de reprendre la main dans sa relation avec l'équipe soignante.

Cette courte étude de cas illustre l'une des modalités selon lesquelles le politique - sous la forme de l'engagement militant - peut s'inviter entre les

3. Revel J., "Présentation », in Revel J. (ed.), Jeux d'échelles. La micro-analyse à l'expérience, Paris, Seuil, 1996, p. 12.

4. Le SED (Sozialistische Einheitspartei Deutschlands, Parti socialiste unifié allemand) est fondé le 21 avril 1946, il est issu de la fusion du parti social-démocrate allemand (SPD, Sozialdemokratische Partei Deutschlands) et du parti communiste allemand (KPD, Kommunistische Partei Deutschlands) de la zone d'occupation soviétique.

5. Pennetier C., Pudal B., "La volonté d'emprise », in Pennetier C., Pudal B. (eds.), Autobiographies, autocritiques, avenx dans le monde communiste, Paris, Belin, 2002, pp. 1539.

6. Dans les années 1960, environ 9 à $10 \%$ de la population de RDA appartient au SED.

7. Voir note 4. 
murs d'un service psychiatrique en RDA et influencer les stratégies mises en œuvre par ses acteurs, notamment quand il s'agit de «patients-camarades ». Cette pénétration du politique dans le quotidien psychiatrique ouvre nombre de questionnements qui portent à la fois sur le fonctionnement interne $\mathrm{du}$ parti communiste, sur les mécanismes inhérents à la société est-allemande mais également sur la valeur heuristique d'un tel observatoire. En RDA comme ailleurs, les services psychiatriques accueillent des individus qui se trouvent, de manière plus ou moins durable, à la marge de la société environnante. De la névrose à la psychose, de la dépression à la schizophrénie, les troubles mentaux traduisent tous un « décrochage » opéré entre l'individu et la société, qu'il se manifeste ponctuellement ou qu'il s'inscrive dans la durée. Cependant, l'expérience sociale de ces individus, si elle prend une forme exacerbée, ne s'inscrit pas moins dans le contexte social et historique dont ils sont issus. En nous penchant sur les «patients-camarades », nous faisons donc le choix d'entrer dans la société de RDA par ses marges, afin d'interroger d'une manière inédite les logiques de pénétration de la société par le politique.

Les services psychiatriques de la clinique psychiatrique et neurologique de la Charité tiendront lieu d'observatoire à cette étude. Comme son nom l'indique, cette clinique, qui fait partie de l'ensemble hospitalier le plus important de RDA, comprend à la fois des services de psychiatrie et des services de neurologie, suivant la tradition allemande qui associe les deux disciplines ${ }^{8}$. Il s'agit d'un établissement urbain, situé à Berlin-Est, non loin de la zone-frontière puis du mur de Berlin érigé en août 1961. Les services psychiatriques y accueillent des patients qui se présentent pour la plupart de façon volontaire, de leur propre initiative ou sur la recommandation d'un médecin. Les diagnostics font généralement état de troubles névrotiques ou d'états dépressifs. Quant aux séjours, ils sont principalement de courte ou de moyenne durée. Dans le cadre de notre étude, nous nous intéressons à la période des années 1960, qui sont marquées par la direction du professeur Karl Leonhard (19571969) et qui voient, à l'échelle de la RDA, l'achèvement des grands bouleversements économiques et sociaux amorcés au cours de la décennie précédente.

Bien que notre étude se concentre sur la situation des «patients-camarades ", il convient d'esquisser un rapide tableau concernant le positionnement politique des médecins, afin d'appréhender le contexte au sein duquel vont se jouer les interactions que nous nous proposons d'analyser. Tout au long de l'existence de la RDA, seule une minorité de médecins appartient au SED ${ }^{9}$. Plusieurs raisons expliquent cette situation, au premier rang desquelles

8. Sur l'histoire de la Charité, voir : Bleker J., Hess V. (eds.), Die Charité. Geschichte eines Krankenhauses, Berlin, Akademieverlag, 2010 ; Herrn R., Hottenrott L. (eds.), Die Charité zwischen Ost und West 1945-1992. Zeitzengen erinnern sich, Berlin, bebra Verlag, 2010. Sur la clinique neurologique et psychiatrique de la Charité, voir : Atzl I., Hess V., Schnalke V., Zeitzengen Charité. Arbeitswelten der Psychiatrischen und Nervenklinik 1940-1999, Münster, LIT Verlag, 2005. Voir également l'article de Sophie Ledebur dans ce numéro.

9. D’après A. Hecht, $16 \%$ des médecins appartiennent au SED en RDA. Hecht A., Die 
on peut citer l'orientation politique plutôt conservatrice qui caractérise cette profession. La restructuration du système de santé d'après les principes du " centralisme démocratique ", la politique vexatoire mise en place à l'encontre des médecins libéraux mais également leur origine sociale constituent des éléments supplémentaires permettant d'expliquer la position plutôt distanciée voire critique de la majorité des médecins vis-à-vis du parti communiste. Il faut noter cependant que le taux d'adhésion au parti est logiquement plus élevé à l'hôpital de la Charité, établissement-vitrine du régime socialiste ${ }^{10}$. Au cours des années 1960, la clinique psychiatrique et neurologique de la Charité dispose d'une cellule du parti ${ }^{11}$. Elle compte une petite vingtaine de membres, ce qui correspond à 9-10\% du personnel 12. Dans les années 1960, entre un quart et un tiers des médecins en fait partie ainsi que la moitié des médecinschefs. Les camarades sont donc plutôt bien représentés sur les plans numérique et hiérarchique, mais leur action idéologique ne semble pas aussi efficace qu'elle devrait l'être, du fait notamment de la personnalité du directeur de la clinique, Karl Leonhard. Malgré la haute fonction qu'il occupe, ce professeur venu de l'Ouest n'appartient pas au parti communiste et s'intéresse davantage aux perspectives scientifiques qu'aux problématiques politiques ${ }^{13}$.

Pour bâtir cette étude, nous nous sommes appuyée sur un corpus d'une dizaine de dossiers de patients appartenant au SED et ayant fréquenté les services psychiatriques de la Charité entre 1959 et $1964{ }^{14}$. Nous avons retenu les dossiers dans lesquels l'appartenance au parti communiste se voit thématisée, qu'elle apparaisse comme un facteur explicatif de l'état de santé du patient, qu'elle soit utilisée par le patient pour mettre en avant son capital politique ou qu'elle influence le processus thérapeutique. Ces dossiers constituent des pièces rares, des singularités au sein des archives de la clinique. Dans notre analyse, ils représentent autant de «cas » dans la mesure où ils « font problème », et offrent ainsi la possibilité de s'interroger autour d'une question commune : celle du croisement et de l'articulation entre une logique politique - liée à l'appartenance au parti communiste - et une logique médicale - liée au statut de patient d'un service psychiatrique. Si l'objectif de ces études de cas

Wissenschaftselite Ostdeutschlands. Feindliche Übernahme oder Integration ?, Leipzig, Verlag Faber \& Faber, 2002, p. 63.

10. Ibid., p. 63. D'après l'auteur, $28 \%$ des médecins de la Charité sont membres du SED en 1979.

11. En RDA, les membres du SED sont organisés sur leur lieu de travail.

12. Landesarchiv Berlin [LAB], C REP 904-225, Nr 13, Einschätzung der ideologischen Situation an der Nervenklinik, 8.12.1964. LAB, C REP 901-01-12, Nr 73, Abschlußbericht der Brigade Innere Kliniken der Charité von Ende Juni bis 10. September 1960, p. 9. LAB, C REP 904225, Nr 3, Rechenschaftsbericht der Parteileitung der Medizinischen Fakultät, 4 mai 1964.

13. Sur l'apolitisme revendiqué des professeurs de médecine en RDA, voir : Jessen R., « Dictature communiste et milieu universitaire. Étude d'histoire sociale des professeurs d'université de la RDA 1945-1961 », Annales. Histoire, Sciences sociales, 53, 1, 1998, pp. 91-117. Sur les professeurs d'université en RDA : Jessen R., Akademische Eliten und kommunistische Diktatur. Die ostdeutsche Hochschullebrer in der Ulbricht-Ära, Göttigen, Vandenhoeck \& Ruprecht, 1999.

14. Je remercie vivement l'Institut d'histoire de la médecine de Berlin, grâce auquel j'ai accès aux archives administratives et médicales de la clinique psychiatrique et neurologique de la Charité. 
n'est pas l'affirmation d'une règle universelle, elles nous permettent de saisir, dans leur singularité, une « autre » expérience du communisme au quotidien. Comme l'ont formulé Jacques Revel et Jean-Claude Passeron, penser par cas consiste à «prendre en compte une situation, [à] en reconstruire les circonstances - les contextes - et [à] les réinsérer ainsi dans une histoire »15. Cette histoire complexe au sein de laquelle nous nous proposons de réinsérer ces " cas ", c'est celle de la société de RDA et de ses enchevêtrements avec le politique.

Environ deux tiers de ces dossiers concernent des hommes, cette distribution s'expliquant notamment par le moindre engagement politique des femmes au sein du SED. Ces individus, âgés de 28 à 55 ans, exercent pour la plupart une activité professionnelle au moment de leur admission en psychiatrie. Les catégories socio-professionnelles représentées dans ce corpus sont diverses. On peut toutefois noter la surreprésentation d'individus exerçant ou ayant exercé une activité en lien avec l'enseignement. Cet état de fait peut s'expliquer d'une part par la surreprésentation des enseignants du secondaire au sein du parti, et d'autre part par la pression idéologique exercée sur cette profession hautement stratégique pour les autorités communistes ${ }^{16}$. Dans leur majorité et d'après leurs dossiers médicaux, ces individus souffrent de névroses ou de troubles névrotiques, comme la plupart des patients admis au sein de ces services.

Ces dossiers médicaux contiennent des documents de nature diverse (pièces médicales et administratives, lettres personnelles, etc.) ainsi qu'une Krankengeschichte (histoire du malade) qui rend compte du séjour du patient de son admission à sa sortie. La Krankengeschichte se divise en deux temps. On y trouve tout d'abord l'anamnèse - du grec mnêsia ("se souvenir ») : il s'agit de la retranscription de renseignements fournis par le patient lui-même ou par l'un de ses proches, sur son passé et sur l'histoire de sa maladie. Elle se poursuit ensuite avec le parcours de soin (Verlanf), qui retrace le séjour au sein des services. Ce sont les médecins qui rédigent ce document, qui permet aux équipes soignante et médicale d'identifier et de suivre ceux dont elles ont la charge. Le rédacteur choisit de faire mention de certains événements ou dires du patient, s'attachant à mettre en lumière ce qui pourrait éventuellement expliquer la maladie diagnostiquée. Ce document soulève de multiples problèmes d'interprétation pour l'historien. Il est difficile de repérer avec certitude les auteurs des différentes parties qui forment la Krankengeschichte. En outre, ce document suit une logique essentiellement démonstrative en lien avec l'élaboration d'un diagnostic médical et il se fait souvent lacunaire ou simplement allusif. Il est donc nécessaire d'être conscient des processus de

15. Passeron J.-C., Revel J., « Penser par cas. Raisonner à partir de singularités », in Passeron J.C., Revel J. (eds.), Penser par cas, Paris, Éditions de l'EHESS, 2005, p. 22.

16. Droit E., Vers un homme nouveau? L'éducation socialiste en RDA (1949-1989), Rennes, PUR, 2009, pp. 169-179. 
sélection, de mise sous silence et de reformulation à l'œuvre dans la production de ce document, ainsi que de sa dimension volontairement démonstrative. Cependant, et c'est ce qui constitue le cœur de notre travail de recherche, la Krankengeschichte peut également receler nombre d'informations particulièrement précieuses pour l'historien de la société.

C'est donc à travers l'analyse de ce matériau original que nous proposons de réfléchir, à partir d'études de cas, aux modalités selon lesquelles, logiques politiques et logiques médicales s'entrecroisent, se recoupent ou s'affrontent, quand des camarades du parti communiste se font patients d'un service psychiatrique, et vice-versa. En concentrant notre réflexion sur les " patientscamarades ", nous tentons de reconstituer la multi-appartenance de ces individus ainsi que la diversité des stratégies sociales élaborées au sein de l'univers psychiatrique. Il s'agit d'une analyse micro-historique qui privilégie l'expérience des acteurs ainsi que leurs interactions sociales, notre propos s'inscrivant dans une perspective socio-historique dans la lignée de l'historiographie française sur la RDA ${ }^{17}$. Au cœur de notre analyse se trouvent les membres du parti communiste, que nous considérons non pas comme une instance qui ferait face à la société mais bien plus comme l'un de ses éléments constitutifs ${ }^{18}$.

\section{Appartenir au parti : un principe de différenciation sociale au sein des ser- vices psychiatriques?}

Si l'appartenance au parti communiste joue un rôle important au sein de la société extérieure, on peut se demander ce qu'il en est dans les murs de ces services psychiatriques. Qu'est-ce qui différencie les «patients-camarades» des autres ? Quelles relations entretiennent représentants du parti et membres de l'équipe médicale ? Et quelles conséquences cela a-t-il sur les stratégies mises en ouvre par les acteurs?

Pour savoir si un patient est membre du parti communiste, il suffit à l'équipe soignante d'ouvrir son dossier médical. En effet, dans la plupart des cas, lorsqu'il arrive à la clinique, le patient se livre à une anamnèse, retranscrite dans son dossier. L'appartenance au parti y semble alors très souvent évoquée de manière spontanée. Lorsqu'il se raconte, le patient mentionne plus ou moins naturellement les activités politiques qui font partie intégrante de sa vie professionnelle et sociale.

17. Voir notamment : Droit E., op.cit. ; Kott S., Le communisme au quotidien, les entreprises d'État dans la société est-allemande, Paris, Belin, 2001 ; Rowell J., Le totalitarisme au concret. Les politiques du logement en RDA, Paris, Economica, 2006 ; Droit E., Kott S. (eds.), Die ostdeutsche Gesellschaft. Eine transnationale Perspektive, Berlin, Links, 2006.

18. Gieseke J., Jessen R., "Die SED in der staatsozialistischen Gesellschaft », in Gieseke J., Wentker H. (eds.), Die Geschichte der SED. Eine Bestandsaufnahme, Berlin, Metropol Verlag, 2011, pp. 16-60 (p. 16). 
Un élément supplémentaire permet parfois de distinguer les patients membres du parti. Ces individus sont directement soumis à une pression disciplinaire qui peut dans certains cas expliquer leur état de santé. Il arrive ainsi que le patient établisse un lien direct entre son malaise ou son mal-être et sa relation au SED. Sans parler de «syndrome » propre aux membres du parti, force est de constater certaines similitudes concernant les peurs, déceptions et angoisses des membres du SED. Plusieurs patients évoquent ainsi les procédures disciplinaires internes au parti qui peuvent déclencher anxiété ou ressentiment. D'après les statuts du SED de 1950, les sanctions disciplinaires internes peuvent frapper : «quiconque ne respecte pas l'unité et la pureté du parti, n'accomplit pas ses décisions, ne respecte pas la démocratie interne au parti, fait un mauvais usage de son statut de membre ou des fonctions qui lui sont déléguées, ne se montre pas digne dans sa vie personnelle d'un membre du parti, enfreint la discipline du parti $» 19$. Les procédures disciplinaires sont discutées et décidées lors des assemblées générales du parti et sont souvent décrites par les patients comme humiliantes voire traumatisantes. Comme l'écrit Michel Christian, à propos des résultats obtenus à partir de sources orales, «le passage devant l'assemblée générale, même pour des faits apparemment anodins, pouvait avoir des conséquences psychologiques profondes ${ }^{20}$.

Certains propos tenus par les patients ou par leurs proches et retranscrits dans les dossiers viennent confirmer ce constat. En 1960, la femme d'un patient n'hésite pas à évoquer «l'assemblée meurtrière » qui s'est tenue dans le cadre d'une procédure disciplinaire visant son mari ${ }^{21}$. Ce dernier occupe un poste de juriste au Ministère des Affaires étrangères depuis 1954. La perte d'un document important entrâne le déclenchement d'une procédure disciplinaire à son encontre au sein du ministère mais également du parti (Parteiverfahren). A cette occasion, il avoue le départ de son père pour l'Ouest, ce qui fragilise d'autant plus sa position qu'il occupe un poste à responsabilité au sein de l'administration étatique. D'après sa femme, suite à cette procédure disciplinaire «il aurait tout remis en question : qu'il était un bon fils, un bon camarade, un bon époux, etc. ». On perçoit ici combien cet épisode ébranle profondément cet individu, et ce dans toutes les dimensions de son identité. Il semble tiraillé entre deux univers contradictoires : d'un côté, sa famille composée de son père parti pour l'Ouest, mais aussi de sa femme qui semble plutôt critique vis-à-vis des procédures internes au SED ; de l'autre, son travail et son appartenance au parti, qui exige de lui qu'il soit un «bon camarade », c'est-à-dire qu'il se dévoue pleinement à la cause communiste. Ce tiraillement semble être à l'origine de cette situation de crise : ces expériences

19. Statuts du SED, 1950.

20. Christian M., «Le parti et la vie privée de ses membres en RDA », Politique, culture, société, $n^{\circ} 7$, janvier-avril 2009.

21. HPAC, M, 337 / 61. 
socialisatrices contradictoires font éclater «l'illusion identitaire de l'unité de soi », ce qui peut expliquer cette totale remise en question ${ }^{22}$. Les procédures disciplinaires, parce qu'elles touchent le cœur de l'identité sociale, peuvent ainsi susciter un ébranlement profond de la personne mise en cause. D'après Yves Cohen, l'une des particularités historiques du communisme serait sa capacité à faire appel, à une échelle inédite, aux ressources du moi ${ }^{23}$. Le moi communiste mobiliserait systématiquement les autres moi distribués dans les divers engagements de la personne. Remettre en cause le capital politique, la loyauté du moi communiste, introduirait ainsi une totale remise en cause du rapport à soi. D'après cette hypothèse, on peut donc interpréter différemment la formulation rapportée par l'épouse : la loyauté politique du patient ayant été remise en question (est-il un bon camarade ?), il en vient à mettre en doute la valeur de ses autres engagements personnels (est-il un bon fils, un bon époux ?).

Les pratiques disciplinaires peuvent ainsi être lourdes de conséquences pour les personnes concernées, qui voient alors tout ou partie de leur identité sociale remise en cause. La patiente A.R., admise à la clinique en février 1960, raconte comment une procédure de ce type, dont elle a été l'objet un an auparavant, a brisé sa confiance envers le SED et explique en partie son état dépressif. Voici ce qui est inscrit dans son dossier, à la date du 27 février 1960 :

"On l'a rayée du parti, le parti ne s'était pas trop posé de questions, il avait rayé des employés. Elle a fait opposition et cela a pris trois mois avant qu'on ne la reprenne dans le parti. Cependant aujourd'hui encore elle n'a pas dépassé ça, elle a perdu confiance, le manque d'un sentiment d'appartenance fait défaut [sic]. On lui dit, que tout est bien oublié, mais elle n'en est pas encore venue à bout. “C’est vraiment dégoûtant!" (pleure)» ${ }^{24}$.

D'après les dires de la patiente, c'est son appartenance à la « classe » des employés qui aurait provoqué son exclusion du parti. Cette accusation fait écho à d'autres attaques qu'elle aurait subies sur son lieu de travail. Au cours de l'entretien, elle explique en effet qu'on lui aurait reproché de provenir de "cercles bourgeois », ainsi que d'avoir eu une aventure avec un homme marié

22. Lahire B., L'homme pluriel. Les ressorts de l'action, Paris, Nathan, 2011 [1998], p. 48.

23. Cohen Y., «La co-construction de la personne et de la bureaucratie : aspects de la subjectivité de Staline et des cadres soviétiques (années 30) », in Studer B., Haumann H. (eds.), Stalinistische Subjekte. Individuum und System in der Sowjetunion und der Komintern, 19291953, Zürich, Chronos Verlag, 2006, pp. 175-196.

24. HPAC, F, 131 / 60. Krankengeschichte, 27.2.60 (Exploration). « Man habe sie aus der Partei gestrichen, die Partei hatte sich das sehr bequem gemacht, hatte Angestellte gestrichen. Sie habe Einspruch dagegen erhoben und es habe 3 Monate gedauert, bis man sie wieder in die Partei aufgenommen hatte. Jedoch sei sie heute noch nicht darüber hinweg, sie habe das Vertrauen verloren, das mangelnde Zugehörigkeitsgefühl fehlt. Man sage ihr, es ist doch alles vergessen, sie habe es aber noch nicht überwunden. "Das ist doch eine Gemeinheit !" (weint)». [Traduction de l'auteur] 
dix ans auparavant. Ce n'est pas tant son comportement en tant que membre du parti qui semble ici être remis en cause mais avant tout son origine et son appartenance sociales. Le capital politique se compose bien de deux éléments qui se combinent l'un avec l'autre : la dimension généalogique de l'individu et sa trajectoire politique ${ }^{25}$. C'est le premier élément qui fait défaut à A. R. dans ce cas précis. Par ailleurs, le capital politique apparaît bien comme un capital particulièrement instable, qui peut être repris puis redonné à nouveau par le parti en tant qu'unique source de légitimation. L'accusation qui frappe cette magistrate d'une cinquantaine d'années, qui a fréquenté l'école du parti avant celle des juges, dont la carrière est intimement liée à son engagement politique, remet en question une large partie de son identité sociale. Elle ne parvient pas à passer outre et ne comprend pas cette procédure d'exclusion qui est finalement suivie, trois mois plus tard, d'une réintégration. L'impossibilité exprimée de faire à nouveau confiance au parti témoigne d'une prise de conscience du caractère arbitraire du processus de légitimation.

Comme le montrent ces exemples, les dossiers de patients peuvent constituer une source d'informations concernant le fonctionnement interne du parti communiste est-allemand et ses répercussions sur les individus. Dans les cas qui ont été exposés, l'appartenance au parti communiste est mise en avant soit par le patient dans son « récit de soi », soit par l'un de ses proches afin d'expliquer son état de santé. On peut constater que les procédures disciplinaires internes au parti revêtent un caractère d'autant plus anxiogène qu'elles remettent en cause une large partie de l'inscription sociale du camarade incriminé. Si l'espace social de la RDA se caractérise par une importance accrue accordée au capital politique, le caractère instable et incertain de ce dernier participe sans aucun doute de l'insécurité psychique des camarades.

\section{Le parti comme source d'informations et partenaire de négociations}

De manière générale, un autre élément permet de distinguer les patients membres du SED : en ce qui les concerne, le parti communiste constitue une instance supplémentaire avec laquelle la clinique peut échanger des informations, voire entamer des procédures de négociations. En effet, quand un patient est identifié comme camarade, et si cela semble nécessaire, la clinique entre bien souvent en contact avec le responsable de l'organisation de base dont il fait partie (le secrétaire du parti). On voit alors apparaittre dans le dossier médical, et suite aux déclarations du patient, de ses proches, voire de ses collègues, la retranscription de discussions entre le médecin et le représentant du parti. Par ce biais, des renseignements concernant le patient circulent entre les services psychiatriques et le parti communiste, qui constitue ainsi une source potentielle d'informations pour les médecins. Les dossiers attestent d'entretiens menés à l'initiative des médecins avec le secrétaire du parti,

25. Pennetier C., Pudal B., op. cit., p. 19. 
d'échanges téléphoniques et de courriers. Ces échanges concernent bien souvent le comportement du patient au travail ou dans la sphère privée. Ils peuvent également avoir trait à certaines procédures disciplinaires le visant ; les médecins ont la possibilité de négocier avec le parti afin de repousser ou d'annuler leur mise en œuvre, en avançant des raisons médicales. Une négociation peut alors s'engager entre le parti en tant qu'instance disciplinaire et la clinique psychiatrique en tant qu'instance de soins. L'exemple du patient T.E., admis en août 1963 à la clinique, est particulièrement parlant. Quelques jours après sa sortie, il revient à la clinique pour une visite de contrôle et est accompagné du secrétaire de son groupe. Celui-ci explique à l'équipe médicale qu'une procédure disciplinaire va être ouverte à l'encontre du patient, on lui reproche son comportement antérieur et notamment d'avoir voulu attenter à ses jours. L'équipe médicale souligne avec insistance qu'à l'époque des faits qui lui sont reprochés, le patient T.E. était «psychiquement malade » et qu'il ne peut donc en être tenu pour responsable. Elle demande au secrétaire du parti de ne pas « humilier par une telle procédure le patient particulièrement sensible » 26. Aux yeux des médecins, la procédure disciplinaire est bien perçue comme une menace pour l'état de santé du patient, dont un des buts affirmés est «l'humiliation » de l'individu mis en cause.

En ce qui concerne le patient Z.R. qui fait plusieurs séjours à la clinique entre janvier et juillet 1963, les négociations semblent particulièrement difficiles à mener. Z.R. est enseignant et membre du parti, son dossier indique qu'il souffre d'une maladie psychosomatique. C'est lors de son deuxième séjour au sein des services psychiatriques qu'apparaît très clairement une divergence entre le SED et l'équipe médicale. Lors de sa réadmission à la clinique, le patient Z.R. évoque la situation conflictuelle dont il souffre :

« Le patient avait l'impression qu'après sa sortie on l'attendait au tournant, pour engager quelque chose contre lui du côté du parti. Des allusions du secrétaire du parti le lui firent déjà remarquer, et il fut convoqué à la mi-mars à l'assemblée générale, où on formula deux accusations contre lui, et où il dut faire une auto-critique. Premièrement il fut mentionné, que l'an dernier, avant son hospitalisation, il avait déclaré devant des collègues que le nouveau directeur adjoint n'était pas très expérimenté sur le plan professionnel, mais était parvenu au poste car il suivait la ligne du parti. Deuxièmement des rumeurs couraient selon lesquelles il aurait raconté il y a trois ans une plaisanterie sur le président du conseil d'Etat. On reprocha au patient un manque de clarté idéologique, on lui enleva ses fonctions de membre de la direction du parti et on lui

26. HPAC, M, 622 / 63. Krankengeschichte, 25.10.63 : « Es wird dem Parteisekretär ausdrücklich erklärt, daß Pat[ient] psychisch krank war und für sein Handeln in dieser Zeit nicht zur Verantwortung gezogen werden kann. Er wird von uns gebeten, den recht empfindlichen Pat[ient] nicht durch so ein Verfahren zu beschämen [...]». [Traduction de l'auteur] 
ordonna de prendre part à toutes les assemblées du parti, séances du conseil pédagogique ou du syndicat, etc. Le patient était agité et dormait mal déjà une semaine avant la convocation annoncée. Environ 3 jours après la discussion, il eut à nouveau des mucosités dans les selles et peu de temps après du sang. [...] En plus de son service à l'école il dut désormais, en raison de l'ordre émis par le parti, participer deux à trois fois par semaine à des réunions, bien qu'il eût été préconisé par la clinique de ne pas le surcharger en dehors de son activité d'enseignement » 27.

Ici encore, c'est une certaine insécurité qui semble caractériser la relation entre ce camarade et le parti. Tout commence par une «impression », alimentée par des «allusions» du secrétaire du parti. Finalement, Z.R. doit passer devant l'assemblée générale et se soumettre au rituel de l'auto-critique, qui prend souvent la forme d'une auto-accusation publique. Le camarade incriminé semble ici bien seul face à une procédure disciplinaire qui s'appuie sur des paroles rapportées par des tiers et qui font référence à des événements $a$ priori sans gravité et qui se seraient passés une ou plusieurs années auparavant. À nouveau, le capital politique apparaît comme particulièrement instable et soumis à des variations sur lesquelles seul le parti peut transiger. Les sanctions prononcées à l'encontre de cet individu sont en totale contradiction avec les préconisations de l'équipe médicale, ce qui va entraîner une procédure de négociation entre la clinique et le parti. Plusieurs semaines après la ré-admission de ce patient à la clinique, une discussion est donc menée, à l'initiative de l'équipe médicale, avec le directeur de l'école et le secrétaire du parti. Un accord semble finalement être conclu : à l'avenir, le patient devra certes prendre part aux réunions publiques du parti mais ne sera chargé d'aucune fonction ou d'un quelconque travail annexe ${ }^{28}$. Dans cet exemple, on voit bien que le patient est soumis à plusieurs sources d'autorité aux intérêts ici divergents. L'autorité du parti ne s'arrête pas aux frontières de la clinique et les médecins doivent être en mesure de négocier avec cette instance de disciplinarisation,

27. HPAC, M, 417 / 63. Krankengeschichte, Wiederaufnahme, 2.3 .63 : «Der Pat[ient] hatte den Eindruck, daß man nach der Entlassung schon auf ihn wartete, um gegen ihn von der Partei aus vorzugehen. Andeutungen des Parteisekretärs liessen ihn das schon merken, und Mitte März wurde er zur Parteiversammlung vorgeladen, wo man 2 Anschuldigungen gegen ihn vorbrachte, und er selbstkritisch Stellung nehmen mußte. Erstens kam zur Sprache, daß er voriges Jahr vor der stat[ionären] Behandlung zu Kollegen geäußert hat, daß der neue stellv[ertretende] Direktor wohl fachlich nicht so versiert sei, aber in die Stellung komme, da er parteilich ausgerichtet sei. Zweitens war ruchbar geworden, daß er vor 3 Jahren einen Witz über den Staatsratsvorsitzenden erzählt habe. Man warf Pat[ient] mangelnde ideologische Klarheit vor, löste ihn als Parteileitungsmitglied ab und befahl ihn, an allen Parteiversammlungen, Sitzungen des Päd[agogischen] Rats oder der Gewerkschaft etc. teilzunehmen. Der Pat[ient] war schon eine Woche vor der angekündigten Vorladung aufgeregt und schlief unruhig. Etwa 3 Tage nach der Aussprache hatte er wieder Schleim im Stuhl und bald darauf Blut. [...] Außer seinem Schuldienst mußte er nun auf Grund des Parteibefehls 2$3 \mathrm{mal}$ in der Woche an Sitzungen teilnehmen, obwohl von der Klinik aus befürwortet war, daß er außerhalb seiner Lehrtätigkeit nicht zu belasten war ». [Traduction de l'auteur]

28. Ibid., Krankengeschichte, Wiederaufnahme, 15.6.63. 
qui poursuit une logique pouvant entrer en contradiction avec les perspectives médicales.

Ces négociations peuvent être d'une toute autre nature quand il s'agit au contraire d'aider le patient à entrer au SED. Ainsi, en 1960, quand un patient évoque ses difficultés professionnelles en lien avec son impossibilité à intégrer le parti, une discussion est organisée quelques jours plus tard, à l'initiative de l'assistante sociale, avec le secrétaire du parti de son lieu de travail ${ }^{29}$. Ce dernier promet alors au patient que sa demande d'adhésion sera examinée dès sa sortie de la clinique. Ici, l'équipe médicale et soignante fait levier afin de faciliter les démarches entreprises par le patient auprès du SED.

Ces exemples montrent dans quelle mesure l'appartenance au parti communiste est susceptible d'entraîner une certaine différenciation sociale parmi les patients des services psychiatriques. Ces «patients-camarades » peuvent manifester des symptômes directement ou partiellement liés à la pression disciplinaire à laquelle ils sont soumis. De plus, dans leur cas, l'équipe médicale dispose d'un interlocuteur supplémentaire, incarné par le secrétaire du parti, ce qui peut influer sur les mécanismes institutionnels internes à la clinique. Dans une autre perspective, le fait d'être « camarade " peut également apparầtre comme une ressource pour ces patients, qui peuvent la mobiliser sciemment et ainsi, dans certaines situations, " reprendre la main » dans le cadre des relations qu'ils entretiennent avec l'équipe médicale.

\section{Être « camarade » avant d'être patient : quand les patients activent leurs capitaux politique et militant 30}

A la différence de l'analyse proposée par Erving Goffman sur le fonctionnement des « institutions totales ", on ne constate pas, pour les patients de la clinique étudiée, de rupture brutale et nette avec leurs "rôles antérieurs » 31. D’après nos sources, les patients trouvent dans leur expérience personnelle, antérieure à leur admission, une ressource non négligeable qui leur permet de négocier avec l'institution. La clinique de la Charité étant une clinique universitaire, la durée d'hospitalisation moyenne est moins longue que dans les autres établissements psychiatriques, ce qui explique la capacité des patients à activer leurs inscriptions sociales et notamment politiques.

29. HPAC, M, 345 / 60, Krankengeschichte, 23.7.60.

30. Au sujet de la distinction à opérer entre capital politique et capital militant, nous nous appuyons sur les travaux de Frédérique Matonti et Frank Poupeau. Le capital militant y est défini comme « les savoirs et les savoir-faire incorporés au fil des expériences politiques », il «s'acquiert [...] dans le champ politique » mais est «susceptible[s] d'être reconverti[s] ailleurs ", il s'agit d' " une forme de capital moins labile que le capital politique [capital symbolique], dans la mesure où celui-ci dépend de l'organisation politique qui le confère à ses membres ». Matonti F., Poupeau F., "Le capital militant. Essai de définition », Actes de la recherche en sciences sociales, $\mathrm{n}^{\circ} 155,2004 / 5$, pp. 4-11. Matonti F., «Crises politiques et reconversions : Mai 68 », Actes de la recherche en sciences sociales, $n^{\circ}$ 158, 2005/3, pp. 4-7. 
Dans le cas de la patiente A.R., qui effectue un séjour de quelques semaines au sein des services psychiatriques entre juillet et septembre 1961, l'appartenance au parti est ainsi mise en avant à son initiative et joue un rôle dans les relations qu'elle entretient avec les autres patients. Au cours de son hospitalisation, cette enseignante de 40 ans se plaint d'avoir été provoquée « politiquement ». Dans son dossier, à la date du 22 août 1961, on peut ainsi lire :

« Melle R. s'est sentie provoquée politiquement par deux autres patients. Après un interrogatoire détaillé, il apparaît cependant que ce sont au contraire les autres patients qui se sont sentis provoqués par son comportement et par des choses, qui n'avaient absolument rien à voir avec la politique $\gg 32$.

Cet événement est rapporté au directeur de la clinique, le professeur Leonhard, qui mène un « interrogatoire très énergique avec les patients que Melle R. désigne comme coupables ». Cependant, il ressort de cet interrogatoire que si certaines déclarations ont été faites, elles n'ont été causées, d'après ces patients, que par l'attitude provocante de l'accusatrice. La patiente n'en reste pourtant pas là. Lors d'échanges téléphoniques avec le secrétaire de sa cellule, elle critique notamment « l'attitude vis-à-vis de la société » des patientes de la clinique ${ }^{33}$. D'une certaine manière, on peut dire que cette patiente use ainsi de son statut de camarade ou plutôt de candidate au parti. En effet, au moment de son admission à la clinique, cette patiente vient d'apprendre qu'elle est acceptée au sein du SED en tant que candidate. Il s'agit d'un statut intermédiaire qui précède l'admission en tant que membre de plein droit, cette période probatoire dure un an et vise notamment à tester les convictions politiques des nouvelles recrues. Cela fait cinq ans que cette femme tente d'entrer au parti, jusqu'alors en vain, en raison notamment de son appartenance passée au parti chrétien-démocrate. A l'été 1961, elle se trouve donc au terme d'un processus de conversion politique entamé il y a plusieurs années. En affirmant son positionnement politique au sein de la clinique, en attaquant les autres patients sur ce terrain, A.R. s'affirme en tant que future camarade du SED, chargée de diffuser le message idéologique des instances dirigeantes. Qu'elle soit politique ou religieuse, la conversion est « un phénomène essentiellement social »; « elle est dès lors qu'elle s'affirme » ${ }^{34}$. Dans

31. Goffman E., Asiles. Études sur la condition sociale des malades mentaux, Paris, Éditions de Minuit, 1968, p. 57.

32. HPAC, F, 432 / 61. Krankengeschichte, 22.8.61 : «Frl. R. fühlte sich durch 2 andere Patienten politisch provoziert. Die genaue Befragung zeigte jedoch, dass sich wiederum die anderen Patienten durch ihr Verhalten provoziert fühlten und zwar durch Dinge, die mit Politik absolut nichts zutun hatten ». [Traduction de l'auteur]

33. Ibid. Krankengeschichte, 4.9.61, Rücksprache mit dem Parteisekretär und stellvertretenden Direktor der Lehrerbildungsanstalt K.

34. Kestel L., La conversion politique. Doriot, le PPF et la question du fascisme français, Paris, Raisons d'agir, 2012, pp. 231-232. 
cette perspective, le choix de son interlocuteur - le secrétaire du parti - prend tout son sens puisqu'il représente la structure sociale qui doit potentiellement accréditer cette conversion. On peut donc supposer que le zèle politique dont fait preuve cette patiente, et qui se manifeste notamment par les plaintes qu'elle transmet au secrétaire du parti, est à mettre en relation avec une volonté de "faire ses preuves ", au terme d'un processus de conversion politique qui touche à sa fin. Elle mobilise, pour le faire sien, un capital politique qui ne lui a pas encore été définitivement conféré par le parti, ainsi qu'un capital militant, qui s'incarne dans les modalités de son engagement et dont on peut peut-être supposer qu'il a été en partie hérité de son expérience politique précédente.

Le fait d'appartenir au SED donne ainsi l'occasion à certains individus de se différencier de la masse des autres patients. Selon le parcours politique et personnel, différentes stratégies sont mises en œuvre. Dans certains cas, cette utilisation du capital politique et/ou militant se manifeste d'une manière particulièrement visible, notamment quand ces individus se font les porte-parole de l'idéologie marxiste-léniniste au sein des services, y faisant circuler les normes et valeurs de la dictature. Voici par exemple ce que l'on peut lire dans le dossier d'un patient, pour l'année 1961 : «Veille aussi à l'ordre dans la chambre et exhorte ses compagnons de chambre à la propreté. Veille aussi à ce qu'ils soient instruits sur le plan idéologique » ${ }^{35}$. D’après le dossier, l'équipe soignante ne semble prendre aucune mesure face à ce comportement. Le silence qui suit la narration de cet événement peut laisser penser que ces actions de «clarification idéologique » sont tolérées au sein des services, ce patient pouvant ainsi jouer le rôle de vecteur «à l'intérieur » de la propagande idéologique omniprésente « à l'extérieur».

Un autre cas vient étayer cette hypothèse et mérite d'être développé plus en détails. En 1964, le patient L.T. est admis au sein de l'unité de psychothérapie de la Charité où il va rester deux mois et demi. D'après les informations qu'il livre sur sa vie personnelle et professionnelle, il s'agit d'un individu particulièrement engagé sur le plan politique qui s'efforce de montrer aux autres « le droit chemin " ${ }^{36}$. À son entrée à la clinique, L.T. ne renonce pas à son engagement, bien au contraire, puisqu'il essaie de convaincre les patients qui ont, d'après lui, «de mauvaises opinions politiques » ${ }^{37}$. Il transpose ainsi une pratique appartenant au champ politique dans une situation sociale située $a$ priori hors-champ. De camarade, il se fait certes patient mais sans abandonner les schèmes d'action inhérents à son expérience politique et qui lui semblent transférables au contexte social des services psychiatriques ${ }^{38}$. Ce faisant, il suit

35. HPAC, M, 465 / 64. Krankengeschichte, Psychisch (non daté) : « Sorgt auch für Ordnung im Zimmer und hält seine Mitpatienten im Zimmer für Sauberkeit an. Sorgt auch dafür, dass diese ideologisch aufgeklärt werden ». [Traduction de l'auteur]

36. HPAC, M, 546 / 64. Krankengeschichte, 12.8.64, Eigene Anamnese, Soziale Entwicklung.

37. Ibid., Krankengeschichte, 12.8.64, Zur Persönlichkeit.

38. Lahire B., op.cit., pp. 42-43 et pp. 93-100. 
à la lettre les «devoirs» des membres du parti, qui ont été précisés dans les statuts de 1963. On peut notamment y lire que chaque membre du parti est tenu "d'expliquer aux masses le sens de la politique et des décisions du parti, de les convaincre de la justesse de la politique du parti, de gagner leur coopération " ${ }^{39}$. Un des principaux devoirs des membres du parti communiste est bien d'œuvrer pour la propagation du message idéologique, et ce quelle que soit la situation sociale dans laquelle ils se trouvent. L'équipe médicale réagit à cette initiative et L.T. est tout d'abord rappelé à l'ordre ; on lui rappelle ainsi qu'il se trouve dans un hôpital, qu'il s'agit de personnes malades, dont le traitement est dans les mains de l'équipe soignante et qu'il doit s'intégrer exactement comme les autres patients. L'équipe soignante met de côté les convictions politiques du patient, son rôle en tant que membre du parti, pour le ramener à sa situation de "patient comme les autres " ${ }^{40}$. La logique médicale et de soin prime alors sur la logique politique. Il y a bien ici un conflit entre des intérêts divergents : d'un côté, les intérêts de l'équipe médicale, qui se veut a priori indifférente aux convictions politiques des patients; de l'autre les intérêts du parti, dont les camarades doivent diffuser le message politique. Cette concurrence des intérêts se cristallise dans la situation de cet individu, tout à la fois patient et camarade. Un peu plus loin dans le dossier, on apprend qu'il lui a finalement été accordé la possibilité de s'exprimer dans le cadre d'un cercle de discussion, il est ajouté qu'il débat avec empressement avec les autres patients quand ceux-ci ont une opinion politique différente de la sienne ${ }^{41}$. Finalement, l'équipe médicale semble avoir fait marche arrière puisqu'elle autorise cet individu à mener des vives discussions politiques avec les autres patients. Cette deuxième étape confirme l'impression de relative tolérance dont le personnel de l'institution psychiatrique fait preuve vis-à-vis d'une certaine forme de propagande idéologique, menée ici à l'initiative des « patientscamarades ».

\section{À «patients-camarades ", « médecins-camarades »}

Au sein des services psychiatriques, les patients ne sont pas les seuls à activer leurs capitaux politique et militant. D'après les sources que nous avons consultées, l'équipe médicale peut également en faire usage dans le cadre notamment du suivi du patient. Les logiques politiques et médicales s'entrecroisant, l'appartenance au SED peut constituer un avantage pour le médecin qui soigne d'autres membres du parti.

En 1960, un patient qui évoque des raisons politiques pour expliquer sa tentative de suicide se voit ainsi proposer de se confier à un médecin-chef membre du SED ${ }^{42}$. L'appartenance politique de ce médecin est mise en avant

39. Statuts du SED, 1963.

40. HPAC, M, 546 / 64, Krankengeschichte, 12.8.64, Zur Persönlichkeit.

41. Ibid., Krankengeschichte, Verlauf (non daté).

42. HPAC, M, 418 / 60. Krankengeschichte, Verlauf, 22.9.60. 
par l'équipe médicale afin de mettre le patient en confiance et de recueillir des informations le concernant.

On retrouve cet enchevêtrement des logiques politiques et médicales dans un autre cas. En 1959, le patient H.G. est admis au sein des services psychiatriques du fait d'un état dépressif. Il appartient au SED depuis 1945 et y a occupé des fonctions à responsabilité (il a notamment exercé la fonction de secrétaire adjoint du parti au sein de son organisation de base). D’après lui, c'est la commission de contrôle du parti qui est à l'initiative de son admission à la clinique. Quelques jours après son hospitalisation, un médecin de la clinique, lui-même « camarade », adresse un courrier au groupe du SED dont fait partie ce patient afin d'obtenir des informations le concernant ${ }^{43}$. Dans cette lettre, le médecin n’évoque pas « le patient G. » ou « monsieur G. ». Il utilise des formules qui laissent de côté la relation patient-soignant pour mettre en avant leur appartenance commune au SED. Il parle ainsi de « notre membre du parti », ou plus loin du « camarade G. ». À la lecture de ce document, rédigé par un médecin sur son patient, on a ainsi l'impression que l'appartenance politique de H.G. prime sur son identité de patient. On peut supposer que le médecin active sciemment ce référentiel identitaire afin d'entrer dans un rapport de connivence avec son interlocuteur et ainsi de recueillir le maximum de renseignements au sujet de son patient. La réponse qu'il reçoit de la part du secrétaire du parti est d'ailleurs adressée au « camarade » Dr. M. ${ }^{44}$. Cet exemple nous montre dans quelle mesure la ligne de partage membres du parti-non membres du parti peut se superposer à la séparation entre patients et soignants. Ces deux exemples remettent à nouveau en cause l'idée d'une identification indifférenciée des patients par le personnel de l'institution et montrent selon quelles modalités l'appartenance au SED peut être utilisée comme une ressource par les médecins.

Au sein des services psychiatriques, une large place est laissée à la parole du patient qui se raconte et évoque bien souvent ses difficultés, qu'elles soient d'ordre personnel ou professionnel. Comme nous l'avons vu précédemment, l'appartenance du médecin au parti peut constituer un critère significatif dans l'établissement de la relation de confiance entre patient et soignant. Dans une logique inverse, le parti lui-même peut intervenir pour empêcher la divulgation d'informations auprès de médecins qui n'appartiennent pas au parti. La non-appartenance au SED est ici perçue comme un critère d'exclusion. Cette intrusion du parti dans la relation patient-soignant se manifeste notamment dans le cas de la patiente H.R., enseignante de 40 ans, qui effectue un séjour d'un mois à la clinique entre février et mars 1961. Dans le cadre des entretiens avec l'équipe médicale, elle évoque notamment les difficultés qu'elle a pu connaître dans sa relation avec le SED. Quelques jours après sa sortie, H.R. se

43. HPAC, M, 18 / 60. Lettre du Dr. M. adressée au camarade H. (secrétaire du parti), datée du 2.2.1959.

44. Ibid., Réponse du secrétaire du parti H. au Dr. M., datée du 10.2.1959. 
présente à nouveau à la clinique dans le cadre du suivi ambulatoire. Elle explique alors avoir essuyé des reproches de la part de l'école du parti suite à sa sortie de la clinique. Voici ce qu'on peut lire dans son dossier, à la date du 26 avril 1961 :

« $\mathrm{M}^{\mathrm{me}} \mathrm{R}$. vient aujourd'hui à notre demande et raconte avec beaucoup de difficultés, qu'en réalité elle ne voulait pas venir. Entretemps, elle avait été renvoyée de l'école du parti, on lui avait retiré son poste de directrice et lors d'un dernier entretien on lui avait dit qu'elle avait fait part d'affaires internes au parti à des personnes qui n'en étaient pas membres (nous) et qu'elle avait ainsi brisé la bonne entente entre Intelligence et parti. Ils lui avaient en outre dit qu'elle ne devait [mot illisible] pas refaire la même erreur et mener à nouveau un entretien avec nous, et que, si elle avait besoin d'un médecin, elle devait se tourner vers le parti, qui lui conseillerait un médecin camarade $» 45$.

Dans cet extrait, il est clairement question du positionnement politique $\mathrm{du}$ médecin : en tant que parteilos (non membre du parti), il ne peut entendre ce qu'un camarade aurait à lui confier, surtout si cela touche aux « affaires internes au parti ». D'après les représentants du SED qui en font le reproche à H.R., un camarade doit toujours se tourner en priorité vers un « médecincamarade ». On retrouve ici une vision dichotomique du monde, qui marque la pensée communiste depuis la redéfinition et la criminalisation du trotskisme dans les années 1930 et qui reste active bien après la mort de Staline ${ }^{46}$. Cette grille de lecture binaire qui sépare les « amis du parti »-incarnés par les camarades - des autres - qualifiés de parteilos - se manifeste clairement ici et empêche H.R. de poursuivre le travail thérapeutique amorcé. La peur de l'Autre se fait double : non seulement celui qui n'appartient pas au parti (en l'occurrence le médecin) ne doit pas entendre ce qu'il s'y passe de peur qu'il utilise ces informations à mauvais escient, mais le camarade lui-même (H.R..) semble susciter de la méfiance de la part des autorités du parti. Le thème de la trahison est récurrent dans l'histoire du communisme et on en a ici une manifestation concrète. Cette «peur de l'Autre » conduit à une certaine solitude des militants qui, à l'image de cette patiente, peuvent faire l'expérience d'une

45. HPAC, F, 106 / 61. Krankengeschichte, ambulante Vorstellung, 26.4.61 : « Heute kommt Frau R. auf unsere Aufforderung und erzählt mit viel Hemmungen, daß sie eigentlich gar nicht kommen wollte. Sie ist inzwischen von der Parteischule verwiesen, ihres Postens als Direktor enthoben und man habe ihr in einer letzten Aussprache gesagt, sie habe parteiinterne Dinge parteilosen Menschen (uns) berichtet und damit das gute Einvernehmen zwischen Intelligenz und Partei gestört. Sie haben ihr weiterhin gesagt, sie solle [mot illisible] nicht weder den Fehler machen und noch einmal eine Aussprache mit uns führen, wenn sie einen Arzt brauche, sollte sie sich an die Partei wenden, die werde ihr dann einen Genossen Arzt empfehlen. » [Traduction de l'auteur]

46. Pennetier C., Pudal B., «La peur de l'autre : vigilance anti-trotskiste et travail sur soi », in Studer B., Haumann H. (eds.), Stalinistische Subjekte. Individuum und System in der Sowjetunion und der Komintern, 1929-1953, Zürich, Chronos Verlag, 2006, pp. 254, 265-267. 
procédure disciplinaire sans logique apparente. La logique politique l'emporte donc ici sur la logique médicale.

Au final, il apparaît que le politique - incarné dans l'appartenance au parti communiste et dans l'engagement militant - peut s'inviter entre les murs des services psychiatriques et en influencer certains mécanismes. Dans le cas des «patients-camarades », l'équipe soignante et médicale dispose d'un interlocuteur supplémentaire, le parti communiste, avec lequel elle peut entrer en contact afin de recueillir des informations sur le patient ou de préparer sa sortie. Les patients eux-mêmes peuvent faire le choix de mettre en avant leur statut de «camarade » dans le cadre des relations qu'ils entretiennent avec les autres patients ou avec le personnel de la clinique. En activant cette inscription dans la sphère politique, ils mettent en concurrence directe deux sources d'autorité aux intérêts divergents : l'institution médicale, pour laquelle ils sont des patients à soigner, et le parti communiste, au sein duquel ils ont le statut de « camarades». Le plus souvent, cette confrontation éventuelle prend la forme d'un croisement des rôles.

Les dossiers de patients que nous avons analysés reflètent des expériences individuelles et détiennent de ce fait un caractère singulier. Ils manifestent des situations dans lesquelles patients et médecins doivent se positionner face à une ligne de partage identitaire de nature politique et qui sépare les « camarades » des «non-camarades ». Si ces situations sont loin d'être quotidiennes, il semble néanmoins particulièrement intéressant de se pencher sur ces «cas-limites » et d'appréhender les patients des services psychiatriques dans leurs interactions avec l'espace social et politique environnant. Se pencher sur l'expérience et le vécu de ces acteurs « en marge de la société » permet non seulement d'approcher une " autre expérience du communisme au quotidien » mais également d'aborder des tensions et des contradictions qui traversent l'ensemble de la société est-allemande. 